\title{
Prospective trial finds nystatin mouthwash effective prophylaxis for pulmonary invasive fungal infections that originate in the throat of patients with hematologic malignancies
}

\author{
R. $\mathrm{HU}^{1}, \mathrm{X} . J \mathrm{JANG}^{1, *}, \mathrm{Y} . \mathrm{WU}^{2, *}$ \\ ${ }^{1}$ Nursing School of Fujian Medical University, 1 Xueyuan Road, Shangjie Town, Minhou County, Fuzhou, Fujian 350108, P.R. China; ${ }^{2}$ Union \\ Hospital Affiliated with Fujian Medical University, 29 Xinquan Road, Fuzhou 350001, Fujian, P.R. China
}

*Correspondence: jiangxy320@126.com and wuyong9195@126.com

Received August 7, 2012 / Accepted October 28, 2012

\begin{abstract}
We investigated the source of fungi in the lungs of patients with hematological malignancies who had invasive pulmonary fungal infections (IPFI). We also conducted a prospective study to evaluate the efficacy of different mouthwash solutions in preventing IPFI in patients with hematologic malignancies. In order to determine the source of fungi in the lungs of 30 patients with hematologic malignancies who had IPFI, we collected samples from sites with suspected fungal infection and used PCR and sequencing for pathogen identification. For the prospective study, we enrolled 158 patients with hematological malignancies who had IPFI and randomly assigned them to one of three mouthwash groups: $1 \%$ nystatin, $2.5 \%$ sodium bicarbonate, or normal saline. Fungal staining and incidence of IPFI, oral fungal infection, and intestinal fungal infection were evaluated. We showed that $96.7 \%$ of the fungi isolated from the throats and the lungs were identical; $76.9 \%$ of the fungi from the lungs and digestive tracts were identical and, $84.6 \%$ of the fungi from the throats and digestive tract were identical. Patients using $1 \%$ nystatin had lower incidence of IPFI (1.6\%) and fungal enteritis (1.6\%) than those using sodium bicarbonate (16.3\% and $14.3 \%)$ or normal saline $(27.7 \%$ and $12.8 \%)$. All treatments had low incidences of oral fungal infections (0 to $4.3 \%)$. Our data showed that fungi originating from mouth and throat cause IPFI. We also showed that use of a prophylactic mouthwash containing $1 \%$ nystatin was effective in preventing IPFI in patients with hematological malignancies.
\end{abstract}

Key words: antifungal agents, hematologic neoplasms/complications, mycoses/prevention and control, pulmonary infection, comorbidity

As high-dose chemotherapies have been widely applied in recent years, in patients with such hematologic malignancies as leukemia, malignant lymphoma, multiple myeloma, and myelodysplastic syndrome have suffered an increasing incidence of fungal infections. One of the most common fungal infections is invasive pulmonary fungal infection (IPFI), which is an important cause of death in patients with hematologic malignancies [1-5]. These patients have inhibited immune function after receiveing radiotherapy and/or chemotherapy. These conditions allow fungi in the mouth to migrate to the lower respiratory tract, which results in fungal infection. We previously showed that risk factors for IPFI include number of days of hospitalization, history of IPFI, number of antibiotics used, sepsis and hypoalbuminemia [6]. Theoretically, the fungi from the lung and throat have sequence homology in IPFI patients, though this homology has not been confirmed in prior published reports. Pretreatment with mouthwash containing appropriate drugs may reduce the fungi in the throat, decreasing the incidence of IPFI. Prophylaxis treatment with antifungals including fluconazole, voriconazole, and posaconazole is recommended [2], but the wide application of these agents is limited by their inevitable side effects, drug interactions, and high costs [7-10].

In China, some physicians have attempted to prevent respiratory tract infections by using sodium bicarbonate mouthwash and airway humidification solutions, though conflicting reports of clinical efficacy have emerged [11-14]. Another possibility is nystatin, which is a broad-spectrum antifungal agent that is effective when focally administered in ointment and lozenge and when used for prevention with nystatin mouthwash [15-17]. When patients receiving hematopoietic stem cell transplantation were given amphotericin 
B or nystatin mouthwash prophylactically, fungi in the throat were markedly reduced, and nystatin had superior taste and patient compliance compared with amphotericin B [18]. Our present study determined the source of fungi in the lung through PCR sequence analyses, and conducted a prospective clinical trial to compare the efficacy of prophylaxis to prevent IPFI through mouthwash solutions with normal saline, $2.5 \%$ sodium bicarbonate, or $1 \%$ nystatin in normal saline.

\section{Materials and methods}

Fungi detection. We recruited 30 IPFI in patients with hematologic malignancies from the Affiliated Union Hospital of Fujian Medical University between August 2010 and January 2011. The patients included 16 men and 14 women, and had a median age of 55 years (range: $19-81$ years). The hematological malignancies were acute leukemia in 13 patients, lymphoma in 9 , multiple myeloma in 5 , myelodysplastic syndrome in 2 , and chronic leukemia in 1.

A total of 75 samples were collected from sites with suspected fungal infections and found to be clinically positive for fungi, including throat swab samples $(n=30)$, sputum samples $(\mathrm{n}=30)$, feces samples $(\mathrm{n}=13)$, a blood sample $(\mathrm{n}=1)$, and a nasal secretion sample $(n=1)$. Notably, one feces sample and one blood sample were missing, so a total of 73 samples were amplified by PCR and sequenced. The single colonies were collected from positive samples and were cultured at $30^{\circ} \mathrm{C}$ for one to two days in Sabouraud's medium, a single colony was collected again in $6 \mathrm{~mL}$ Sabouraud's liquid medium, cultured one to two days at $30^{\circ} \mathrm{C}$ with continuous shaking, and then centrifuged at $4000 \mathrm{~g}$ for 2 minutes. The supernatant was removed, and the fungi were collected and weighed.

The fungal DNA was extracted with the E.Z.N.A. ${ }^{\circledR}$ Fungal DNA Mini Kit (Omega Bio-tek, Inc., Norcross, GA) by mixing $100 \mathrm{mg}$ of fungi, $600 \mu \mathrm{L}$ FG1 buffer, and $500 \mathrm{mg}$ glass beads with a diameter of $425-600 \mu \mathrm{m}$ (Sigma, St. Louis, MO). Then, the mix was intensively vortexed for 30 minutes until the solution was clear, and subsequent procedures were carried out according to the manufacturer's instructions.

The 26s rDNA internal transcribed spacer (ITS) sequence of the fungi was PCR amplified using the Fungi Identification PCR Kit (Code No.D317; TaKaRa Bio Inc., Shiga, Japan). PCR amplification was performed for strain/species identification and PCR products were identified after electrophoresis of 5 $\mu \mathrm{L}$ of the products on a $3 \%$ agarose gel. The PCR products were recycled with the Agarose Gel DNA Purification Kit Ver.2.0 (Code No. DV805A; TaKaRa). The sequence of the 26S rDNA ITS was determined with the BigDye Terminator V3.1 Cycle Sequencing Kit (Invitrogen, Carlsbad, CA), and the data was analyzed with the Sequencer 4.8 software. The fungal strains were identified by comparing the sequences of the fungal DNA to those in the National Center of Biotechnology Information.

Prospective clinical trial. The clinical trial was approved by the ethical review board of the Affiliated Union Hospital of Fujian Medical University before any patients enrolled, and informed consent was obtained from the patients. $\mathrm{Pa}$ tients could be enrolled in the study if they did not receive hematopoietic stem cell transplantation but had hematologic malignancies including acute leukemia, chronic leukemia, lymphoma, multiple myeloma and myelodysplastic syndrome; they were hospitalized in the Department of Hematology of Affiliated Union Hospital of Fujian Medical University between 1 January 2010 and 31 December 2010; they were not transferred from another hospital or other departments of the Affiliated Union Hospital; they had no history of IPFI; and their expected hospital stay was longer than 9 days. Patients were excluded from the study if they were diagnosed with IPFI within $48 \mathrm{~h}$ after admission (1); their hospital stay was $\leq 8$ days; they were administered mouthwash for less than 8 days; they were also administered oral or venous anti-fungal agents to prevent fungal infections; or they were not compliant to the treatment.

The enrolled patients were randomly assigned to one of three mouthwash treatment groups: normal saline, $2.5 \%$ sodium bicarbonate made with $125 \mathrm{ml} \mathrm{5 \%}$ sodium bicarbonate and $125 \mathrm{ml}$ normal saline, or $1 \%$ nystatin in $250 \mathrm{ml}$ normal saline $\left(250 \times 10^{4} \mathrm{U} /\right.$ tablet; Shanghai Sine Pharmaceutical Co., Ltd). Each mouthwash was made fresh daily. The mouthwash was administered from the day of admission to the day of discharge. Each patient gargled with mouthwash after each meal and at bedtime for at least three times daily and at least 15 minutes each time. When a patient developed an oral or pulmonary fungal infection, other mouthwash was introduced, based on the advice of his or her physician.

The patients were evaluated based on the incidence of IPFI, the strain of fungi, the incidence of oral fungal infection, and the incidence of intestinal fungal infection. While the patients were hospitalized, samples were collected by specialized nurses from the throat and sputum (for patients with expectoration) at least three times, with the collections occurring before mouth was administered on the day of admission, on day 8 , and on the day before discharge. Sputum samples were collected early in the morning, before the patients ate or drank. To produce a sample of sputum, patients were asked to breathe deeply, and then to cough deeply. The first sputum sample was discarded, and the second sample was collected for fungal culture. Since patients with hematological malignancies are immunocompromised, fibrobronchoscopy is not appropriate for obtaining sputum samples.

Patients who developed a fever had blood collected for fungal culture, and patients who developed diarrhea had feces collected for fungal culture. Fungi were cultured from these samples, and the frequency of fungal culture was increased when necessary.

Statistical analysis. Demographic and basic characteristics of three treatment groups were compared by the chi-square test and by Fisher's exact test for categorical variables. The data are presented as number (n) or percentage (\%). Because 
normality could not be assumed for hospitalization, it is presented as a median with interquartile range according to the Kruskal-Wallis test. All statistical assessments were two-sided and evaluated as significant when $P<0.05$. Statistical analyses were performed with SPSS version 15.0 software (SPSS Inc., Chicago, IL, USA).

\section{Results}

Fungi detection. All our study patients were confirmed as having IPFI, based on clinical symptoms and culture (all > 100 $\mathrm{cfu}$ ). When fungal strains were identified (Table 1) and their genes were sequenced (Table 2), 96.7\% (29/30) of the fungi from the throats and lungs of the IPFI patients with hematologic malignancies had identical $26 \mathrm{~S}$ rDNA ITS sequences. Thus, the fungi that resulted in throat infections were the same as those leading to pulmonary infections in the same patients. Among these 29 patients, eight different strains of Candida albicans were found in 25 patients, while the four remaining patients were infected with Candida krusei, Candida glabrata, Candida tropicalis, or Candida portugal. Additionally, one patient had Candida albicans identified in samples from both the throat and feces, though Aspergillus flavus with identical 26S rDNA ITS sequences were found in the sputum and nasal secretions samples.

When fungal samples from the throat and digestive tracts of IPFI patients with hematologic malignancies were compared, $84.6 \%(11 / 13)$ had identical 26S rDNA ITS sequences. Also, fungi samples from the lung and digestive tracts had identical $26 \mathrm{~S}$ rDNA ITS sequences in $76.9 \%(10 / 13)$ of samples. Figure 1 shows the sequencing chromatograms from the 8 strains of Candida albicans. All the DNA products were from isolated,

Table 1. Sequences of $26 \mathrm{~S}$ rDNA ITS in patients with IPFI

\begin{tabular}{|c|c|c|c|c|}
\hline Patient No & Source of sample & Genus of fungi & Strain & Gene No. \\
\hline 1 & Throat, sputum & Candida albicans & ATCC48130 & GU319987.1 \\
\hline 2 & Throat, sputum, feces & Candida albicans & ST3477-03 & AY939789.1 \\
\hline \multirow[t]{2}{*}{3} & Throat, sputum & Candida albicans & WM10.105 & HQ014723.1 \\
\hline & Feces & Candida krusei & Undetected & \\
\hline 4 & Throat, sputum & Candida albicans & 02-201144 6828 & FJ159658.1 \\
\hline 5 & Throat, sputum, feces & Candida albicans & $02-2011446828$ & FJ159658.1 \\
\hline \multirow[t]{2}{*}{6} & Throat, sputum & Candida albicans & 02-200956 6809 & FJ195646.1 \\
\hline & Blood & Penicillium & Undetected & \\
\hline 7 & Throat, sputum, feces & Candida albicans & ATCC14503 & GU319991.1 \\
\hline 8 & Throat, sputum, feces & Candida albicans & 02-200956 6809 & FJ159647.1 \\
\hline 9 & Throat, sputum, feces & Candida albicans & ATCC14503 & GU319991.1 \\
\hline 10 & Throat, sputum & Candida krusei & KDLYS101 & HM771638.1 \\
\hline 11 & Throat, sputum, feces & Candida albicans & ATCC14503 & GU319991.1 \\
\hline 12 & Throat, sputum & Candida albicans & 02-201144 6828 & FJ159658.1 \\
\hline 13 & Throat, sputum & Candida albicans & IFM49030 & AB369915.1 \\
\hline 14 & Throat, sputum & Candida albicans & ATCC48130 & GU319987.1 \\
\hline 15 & Throat, sputum & Candida albicans & ATCC48130 & GU319987.1 \\
\hline 16 & Throat, sputum & Candida albicans & $02-2002706410$ & FJ159652.1 \\
\hline 17 & Throat, sputum & Candida albicans & $02-2011446828$ & FJ159658.1 \\
\hline 18 & Throat, sputum & Candida albicans & 02-200956 6809 & FJ159647.1 \\
\hline 19 & Throat, sputum & Candida albicans & 02-201144 6828 & FJ159658.1 \\
\hline 20 & Throat, sputum & Candida albicans & WM10.94 & HQ014713.1 \\
\hline 21 & Throat, sputum & Candida albicans & ATCC14503 & GU319991.1 \\
\hline 22 & Throat, sputum & Candida glabrata & KW284 & FN652302.1 \\
\hline 23 & Throat, sputum, feces & Candida albicans & 02-200956 6809 & FJ159647.1 \\
\hline 24 & Throat, sputum, feces & Candida albicans & ATCC14503 & GU319991.1 \\
\hline \multirow[t]{2}{*}{25} & Throat, sputum & Candida tropicalis & WM1045 & EF568041.1 \\
\hline & Feces & Portugal candida & CBS10625 & EU149777.1 \\
\hline 26 & Sputum & Portugal candida & CBS10625 & EU149777.1 \\
\hline \multirow[t]{2}{*}{27} & Sputum, feces & Candida albicans & ST3477-03 & AY939789.1 \\
\hline & Sputum, nasal secretion & Aflatoxin & WM10.84 & HQ014704.1 \\
\hline 28 & Throat, sputum, feces & Candida albicans & ST3477-03 & AY939789.1 \\
\hline 29 & Throat, sputum & Candida albicans & 02-201144 6828 & FJ159658.1 \\
\hline 30 & Throat, sputum, feces & Candida albicans & WM10.105 & HQ014723.1 \\
\hline
\end{tabular}



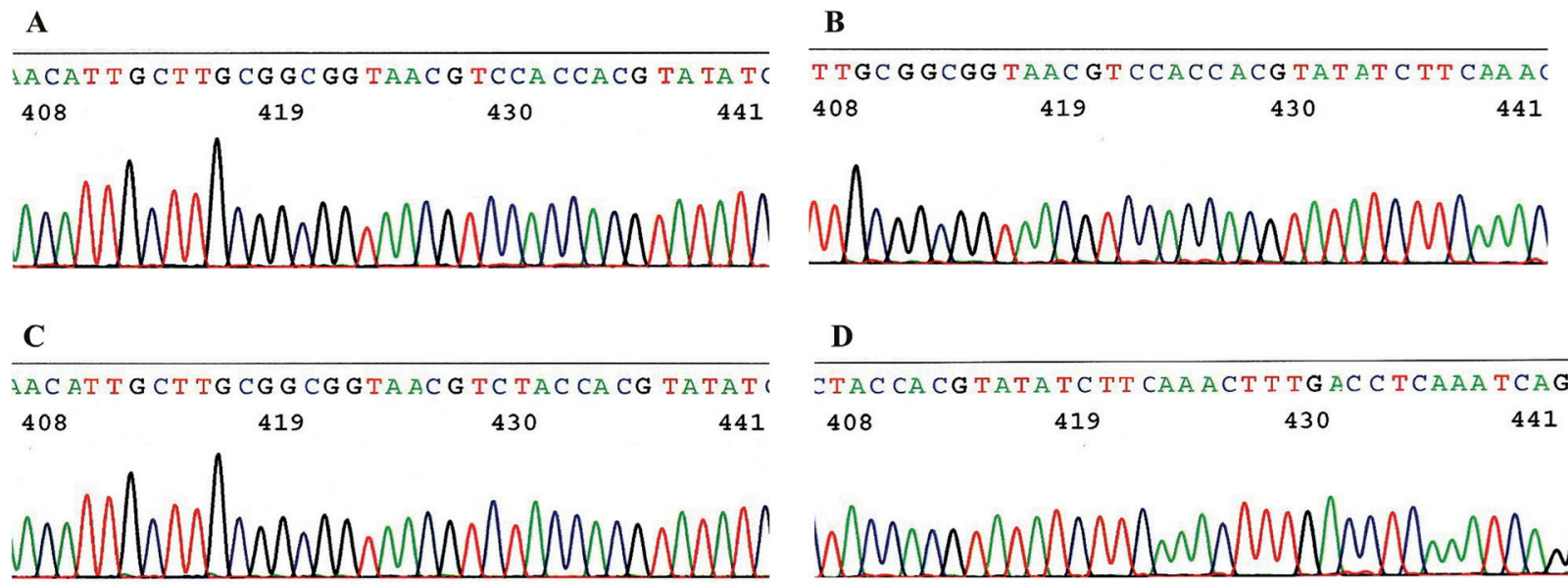

$\mathbf{E}$

IA CT TAATAGT CAAAACT T T CAACAACG A TCTCTTGG

133

144

155

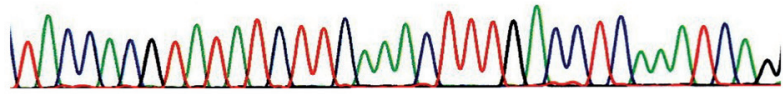

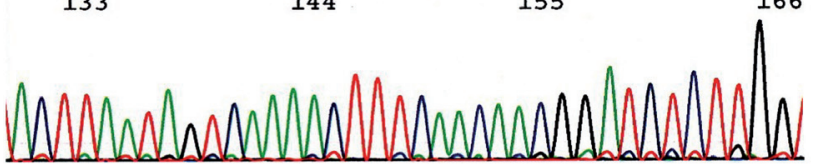

F1

АACT TACAACCAAT TT TTTAT TAACT TGTCACACCAG.

100

111

122

А АCАTTG CT TGCG G CG TA AC T TCAC CA CG IA TA T

$\begin{array}{llll}408 & 419 & 430 & 441\end{array}$

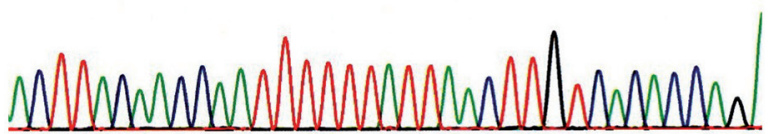

G1

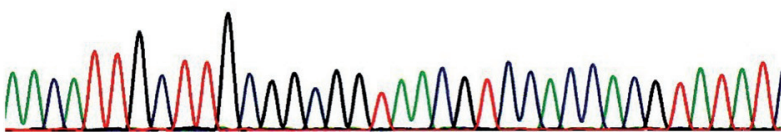

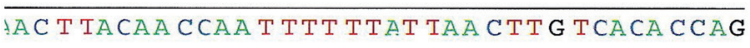

$89 \quad 100 \quad 111 \quad 122$

G2

AA CAT TG CT TG CG G CG TA ACG TC TACCACG TA TA T

$\begin{array}{lll}408 & 419 & 430\end{array}$

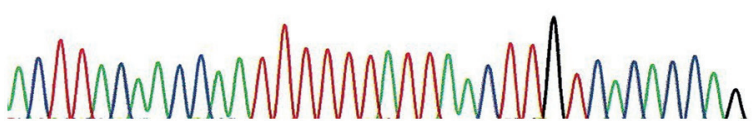

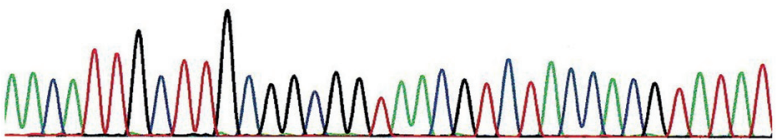

\section{H}

1 A CAT TG T TG C GGCGGTAG CG TCTAC CACG TA TAT

408

419

430

441

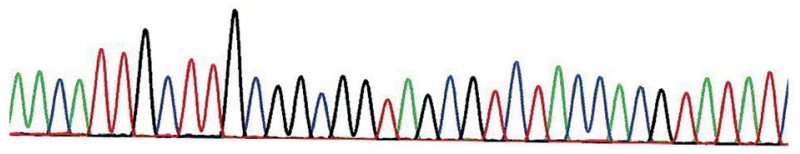

Figure 1. Representative Sequencing Chromatograms of Eight Strains of Candida albicans.

A: Patient No. 1, 14, 15, 20; 431:C (T)

B: Patient No. 2, 27, 28; 414:T (C)

C: Patient No. 3, 30; 431:T (C)

D: Patient No. 4, 5, 12, 17, 19, 29; 424:C (T)

E: Patient No. 6, 8, 18, 23; 132:T (insertion)

F: Patient No. 7, 9, 11, 21, 24; 108:T(C); 431:C(T)

G: Patient No. 13; 108:T(C); 431:T(C)

H: Patient No. 16; 426:G(A) 
single clones and PCR fragments were sequenced directly, with no additional cloning steps.

Prospective clinical trial. A total of 158 patients, enrolled between January 1, 2010 and December 31, 2010, were randomly assigned into three groups (Table 3 ), which were treated with normal saline $(n=47), 2.5 \%$ sodium bicarbonate $(n=49)$, or $1 \%$ nystatin $(n=62)$. The enrolled patients included 109 men and 49 women, who had a mean age of $43.3 \pm 16.9$ years (range: 16-84 years). Among these patients, 102 had acute leukemia, 6 had chronic leukemia, 26 had lymphoma, 15 had multiple myeloma, and 9 had myelodysplastic syndrome. The three groups did not have significant differences in age, gender, type of hematological malignancy, complication, or duration of hospitalization $(P>0.05)$.

The three mouthwash treatments had significant differences in their incidences of IPFI and fungal enteritis $(P<0.05)$. The patients using the nystatin mouthwash had lower incidences of IPFI (1.6\%), enteritis (1.6\%), and oral fungal infections (none) than the normal saline $(27.7 \%, 12.8 \%$, and $4.3 \%$, respectively)
Table 2. Gene polymorphisms of eight strains of Candida albicans

\begin{tabular}{lll}
\hline Patient No & Strain & Characteristic base \\
\hline $1,14,15,20$ & ATCC 48130 & $431: \mathrm{C}(\mathrm{T})$ \\
$2,27,28$ & ST $3477-03$ & $414: \mathrm{T}(\mathrm{C})$ \\
3,30 & WM 10.105 & $431: \mathrm{T}(\mathrm{C})$ \\
$4,5,12,17,19,29$ & $02-2011446828$ & $424: \mathrm{C}(\mathrm{T})$ \\
$6,8,18,23$ & $02-2009566809$ & $132: \mathrm{T}($ insertion) \\
$7,9,11,21,24$ & ATCC 14503 & $108: \mathrm{T}(\mathrm{C}) ; 431: \mathrm{C}(\mathrm{T})$ \\
13 & IFM 49030 & $108: \mathrm{T}(\mathrm{C}) ; 431: \mathrm{T}(\mathrm{C})$ \\
16 & $02-2002706410$ & $426: \mathrm{G}(\mathrm{A})$ \\
\hline
\end{tabular}

or sodium bicarbonate solution $(16.3 \%, 14.3 \%$, and $2.0 \%$, respectively) mouthwash groups (Table 4 ). However, the normal saline and sodium bicarbonate groups had similar incidences of fungal infections. The oral fungal infection incidences did not have significant differences between the three treatment groups.

Table 3. Patients demographics and characteristics among the three mouth rinse groups $(\mathbf{n}=\mathbf{1 5 8})$

\begin{tabular}{|c|c|c|c|c|}
\hline & $\begin{array}{l}\text { Normal saline solution } \\
\qquad(\mathrm{n}=47)\end{array}$ & $\begin{array}{l}\text { Sodium bicarbonate solution } \\
\qquad(\mathrm{n}=49)\end{array}$ & $\begin{array}{l}\text { Nystatin solution } \\
\quad(\mathrm{n}=62)\end{array}$ & $P$ \\
\hline Age, $n(\%)^{1}$ & & & & 0.294 \\
\hline$<=45$ years & $25(53.2)$ & $29(59.2)$ & $39(62.9)$ & \\
\hline $46-59$ years & $6(12.8)$ & $11(22.4)$ & $11(17.7)$ & \\
\hline$>=60$ years & $16(34.0)$ & $9(18.4)$ & $12(19.4)$ & \\
\hline Gender, $\mathrm{n}(\%)^{1}$ & & & & 0.523 \\
\hline Male & $31(66.0)$ & $32(65.3)$ & $46(74.2)$ & \\
\hline Female & $16(34.0)$ & $17(34.7)$ & $16(25.8)$ & \\
\hline Type of disease, $n(\%)^{2}$ & & & & 0.742 \\
\hline Acute leukemia & $27(57.4)$ & $33(67.3)$ & $42(67.7)$ & \\
\hline Chronic leukemia & $4(8.5)$ & $1(2.0)$ & $1(1.6)$ & \\
\hline Lymphomas & $9(19.1)$ & $8(16.3)$ & $9(14.5)$ & \\
\hline Multiple myeloma & $5(10.6)$ & $5(10.2)$ & $5(8.1)$ & \\
\hline Myelodysplastic syndrome & $2(4.3)$ & $2(4.1)$ & $5(8.1)$ & \\
\hline \multicolumn{5}{|l|}{ Complication, $\mathrm{n}(\%)^{1}$} \\
\hline Hypoproteinemia & $26(55.3)$ & $24(49.0)$ & $32(51.6)$ & 0.823 \\
\hline Upper respiratory tract infection & $9(19.1)$ & $13(26.5)$ & $12(19.4)$ & 0.589 \\
\hline Hospitalization (days) $)^{3}$ & $10(9,17)$ & $12(10,18)$ & $13(9,19)$ & 0.539 \\
\hline
\end{tabular}

$P$-values were determined via Chi-square test ${ }^{1}$, Fisher's exact test ${ }^{2}$, and Kruskal Wallis test ${ }^{3}$.

Values are presented as number (percentage) ${ }^{1,2}$, and median (interquartile) ${ }^{3}$.

Table 4. The fungal infections after 3 treatments $(n=158)$

\begin{tabular}{|c|c|c|c|c|}
\hline & $\begin{array}{l}\text { Normal saline solution } \\
\qquad(\mathrm{n}=47)\end{array}$ & $\begin{array}{l}\text { Sodium bicarbonate solution } \\
\qquad(\mathrm{n}=49)\end{array}$ & $\begin{array}{l}\text { Nystatin solution } \\
\quad(\mathrm{n}=62)\end{array}$ & $P$ \\
\hline Invasive pulmonary fungal infection & $13(27.7 \%)$ & $8(16.3 \%)$ & $1(1.6 \%)^{\mathrm{ab}}$ & $<0.001^{*}$ \\
\hline Fungal enteritis & $6(12.8 \%)$ & $7(14.3 \%)$ & $1(1.6 \%)^{\mathrm{ab}}$ & $0.026^{*}$ \\
\hline Oral fungal infection & $2(4.3 \%)$ & $1(2.0 \%)$ & $0(0.0)$ & 0.197 \\
\hline
\end{tabular}

${ }^{*}$ Indicates a statistically significant difference among the three groups, $P<0.05$.

a Indicates a statistically significant difference between the indicated treatment group and the normal saline group.

${ }^{b}$ Indicates a statistically significant difference between the sodium bicarbonate and the nystatin mouthwash groups.

$P$-values were determined via Fisher's exact test 
Only one patient in the nystatin group was positive for a fungus (Aspergillus) in the sputum. Among the patients receiving the normal saline mouthwash, 13 were diagnosed with IPFI, and 9 of the patients were clinically diagnosed with IPFI. Of these 9, 7 were positive for Candida albicans and 2 for Candida tropicalis, while 4 had suspected IPFI. Among the patients receiving the sodium bicarbonate mouthwash, 8 were diagnosed with IPFI. Of these 8 , one was positive for Candida glabrata in the blood, 3 were positive for Candida albicans, 2 were positive for Aspergillus, and 2 had suspected IPFI.

\section{Discussion}

Our study first applied PCR to amplify the $26 \mathrm{~S}$ rDNA ITS sequences, and found that $96.7 \%$ of the fungi isolated from the throats and lungs of patients with hematological malignancies with IPFI were identical, along with $76.9 \%$ of fungi from the lungs and digestive tracts and $84.6 \%$ of the fungi from the throat and digestive tract. Patients with hematological malignancies have compromised immune function, which is further impacted by radiotherapy and chemotherapy treatments. Our study patients showed no significant differences in the risk factors for IPFI which we previously identified [6]. It is also important to note that all the hospitalized patients in this study had a similar diet and performed regular brushing with similar toothbrushes. All the patients used a mouth rinse solution after brushing.

The high sequence homologies reported here support the concept that fungi localizing in the mouth may enter the lower respiratory tract and cause infection there. The Candida found in the lungs mainly migrates from the throat, so enhancing oral care may help to prevent pulmonary infection by Candida. The predominance of Candida albicans that we observed agrees with reports that Candida albicans is the most prevalent etiological agent [2].

We focused on the internal transcribed spacer (ITS) of the rDNA. Comparing rDNA, which is the gene encoding ribosomal RNA, has become an ideal and reliable method to identify pathogen strains [19], and the ITS region is a non-encoding region of the rDNA that is less affected by environmental factors and rapidly evolves compared with the encoding regions. The two regions of ITS, ITS1 and ITS2, are located between 5.8S rDNA and 26S rDNA and between 5.8S rDNA and 26S rDNA. Because the $26 \mathrm{~S}$ rDNA has a variation rate of $<1 \%$ in the same species and $>1 \%$ among different species of yeast [20-22], it is not widely used to clinically identify pathogens. However, the ITS sequences are highly conserved among different strains but significantly different among different fungi and characterized by polymorphism in sequence. These properties allow the ITS region to provide rich information on mycology, and make it suitable for identifying strains with close phylogenetic relationships $[23,24]$.

The second part of our study compared three mouthwash solutions from patients with hematological malignancies, and found that patients using $1 \%$ nystatin mouthwash had a significantly lower incidence of IPFI (1.6\%) and fungal enteritis (1.6\%) than those using sodium bicarbonate $(16.3 \%$ and $14.3 \%)$ or normal saline $(27.7 \%$ and $12.8 \%)$. All three mouthwash treatments had low incidences of oral fungal infections, ranging from zero to $4.3 \%$. Nystatin, a broad spectrum anti-fungal agent and enhancer of membrane permeability, is efficacious in treating oral fungal infections [15-17, 25], but there is no consensus on the concentration of nystatin. We chose $1 \%$ nystatin for our study. Though $0.25 \%$ was suggested as the lowest effective concentration for oral fungal infections [26], on the other side the concentration of $0.25 \%$ nystatin has been suggested as too low to achieve therapeutic efficacy [27], and $0.4 \%$ was ineffective [28]. Nystatin is not absorbed after focal administration, and increasing the dose of nystatin does not affect its safety.

Our study has several limitations. We did not investigate the influence of bacterial infection, and we would not suggest mouthwash to prevent bacterial infections in the clinic. Additionally, nystatin mouthwash is not available commercially, and it must be freshly prepared and used within 24 hours, since it lacks stability at room temperature [14]. Further, we did not investigate the combination of nystatin and sodium bicarbonate to prevent IPFI, which should be studied in the future. Since sodium bicarbonate is alkaline, it can increase the oral $\mathrm{pH}$ to suppress fungal growth.

Overall, we have determined that most fungi causing IPFI originate in the mouth and throat. When we targeted these fungi with three mouthwash solutions, we found that $1 \%$ nystatin significantly reduced the incidence of IPFI and fungal enteritis, more effectively than $2.5 \%$ sodium bicarbonate or normal saline. Though some antifungal agents, including itraconazole and fluconazole, are approved to prevent IPFI [1, 28,29 ], their inevitable side effects, drug interactions, and high costs significantly limit the wide application of these drugs [710]. Nystatin mouthwash may provide an important alternative for use in the clinic to prevent IPFI in a timely manner.

Acknowledgements: Kathy Boltz $\mathrm{PhD}$ of MedCom Asia provided professional English-language writing assistance. This study was supported by Fujian Medical university Youth Fund, \#2010 MP020.

\section{References}

[1] Editorial Committee of Journal of Internal Medicine. Criteria for diagnosis and treatment of invasive pulmonary fungal infections (draft). J Internal Med 2006; 45: 697-700.

[3] HAHN-AST C, GLASMACHER A, MÜCKTER S, SCHMITZ A, KRAEMER A et al. Overall survival and fungal infectionrelated mortality in patients with invasive fungal infection and neutropenia after myelosuppressive chemotherapy in a tertiary care centre from 1995 to 2006. J Antimicrob Chemother 2010; 65: 761. http://dx.doi.org/10.1093/jac/dkp507

[4] KONTOYIANNIS DP, MARR KA, PARK BJ, ALEXANDER $\mathrm{BD}, \mathrm{ANAISSIE}$ EJ et al. Prospective surveillance for invasive fungal infections in hematopoietic stem cell transplant re- 
cipients, 2001-2006: overview of the Transplant-Associated Infection Surveillance Network (TRANSNET) Database. Clin Infect Dis 2010; 50: 1091-1100. http://dx.doi.org/10.1086/ $\underline{651263}$

[5] RUHNKE M, BOHME A, BUCHHEIDT D, CORNELY O, DONHUIJSEN K et al. Diagnosis of invasive fungal infections in hematology and oncology-guidelines from the Infectious Diseases Working Party in Haematology and Oncology of the German Society for Haematology and Oncology (AGIHO). Ann Oncol, 2011.

[6] HU R, WU W, JIANG X. Risk factors for for invasive pulmonary fungal infection in patients with hematological malignancies not receiving hematopoietic stem cell transplant. Neoplasma 2012; 59: 669-675. http://dx.doi.org/10.4149/ neo 2012085

[7] CORNELY OA, MAERTENS J, WINSTON DJ, PERFECT J, ULLMANN AJ et al. Posaconazole vs. fluconazole or itraconazole prophylaxis in patients with neutropenia. $\mathrm{N}$ Engl J Med 2007; 356: 348-359. http://dx.doi.org/10.1056/ NEJMoa061094

[8] ROBENSHTOK E, GAFTER-GVILI A, GOLDBERG E, WEINBERGER M, YESHURUN M et al. Antifungal prophylaxis in cancer patients after chemotherapy or hematopoietic stem-cell transplantation: systematic review and meta-analysis. J Clin Oncol 2007; 25: 5471-5489. http://dx.doi. org/10.1200/JCO.2007.12.3851

[9] SCHONFELD W, WANG CHENG J, TONG KB, SEIFELDIN R. Cost-effectiveness analysis of antifungal prophylaxis in patients undergoing hematopoietic stem cell transplantation. Clin Ther 2008; 30: 964-973. http://dx.doi.org/10.1016/j.clint hera.2008.04.020

[10] GOLAN Y, WOLF MP, PAUKER SG, WONG JB, HADLEY S. Empirical anti-Candida therapy among selected patients in the intensive care unit: a cost-effectiveness analysis. Ann Intern Med 2005; 143: 857-869.

[11] CHEN J, LU W. Oral care with 3\% sodium bicarbonate for prevention of respiratory fungal infection. Chin Clin Med: Drugs and Clinic 2006; 8: 62.

[12] CHEN S, YAN Q, HUANG F. Preventive effect of sodium bicarbonate on pulmonary fungal infection in patients undergoing tracheotomy. J Xiangnan University Med Sci 2006; 8: 55-56.

[13] HE S, WANG X, XIE J. Application of sodium bicarbonate for prevention of fungal infection of lower respiratory tract in patients undergoing mechanical ventilation. Clin Internal Med 2006; 23: 425-426.

[14] SHI Y, LIU F, GANG T. Comparison of preventive effect of two airway humidification solutions on pulmonary fungal infection. Modern Nursing 2008; 14: 270.

[15] GROESCHKE J, SOLASSOL I, BRESSOLLE F, PINGUET F. Stability of amphotericin B and nystatin in antifungal mouthrinses containing sodium hydrogen carbonate. J Pharm Biomed Anal 2006; 42: 362-366. http://dx.doi.org/10.1016/ j.jpba.2006.04.011

[16] LOPEZ-MARTINEZ R. Candidosis, a new challenge. Clinics in Dermatol 2010; 28: 178-184. http://dx.doi.org/10.1016/ j.clindermatol.2009.12.014
[17] BRITO GN, INOCENCIO AC, QUERIDO SM, JORGE AO, KOGA-ITO CY. In vitro antifungal susceptibility of Candida spp. oral isolates from HIV-positive patients and control individuals. Braz Oral Res 2011; 25: 28-33. http://dx.doi. org/10.1590/S1806-83242011005000001

[18] EPSTEIN JB, TRUELOVE EL, HANSON-HUGGINS K, MANCL LA, CHEN A et al. Topical polyene antifungals in hematopoietic cell transplant patients: tolerability and efficacy. Support Care Cancer 2004; 12: 517-525.

[19] KHOT PD, KO DL, FREDRICKS DN. Sequencing and analysis of fungal rRNA operons for development of broad-range fungal PCR assays. Appl Environ Microbiol 2009; 75: 1559-1565. http://dx.doi.org/10.1128/AEM.02383-08

[20] KURTZMAN CP, ROBNETT CJ. Identification and phylogeny of ascomycetous yeasts from analysis of nuclear large subunit (26S) ribosomal DNA partial sequences. Antonie Van Leeuwenhoek 1998; 73: 331-371. http://dx.doi.org/10.1023/ A:1001761008817

[21] KURTZMAN CP, ROBNETT CJ. Identification of clinically important ascomycetous yeasts based on nucleotide divergence in the 5 ' end of the large-subunit (26S) ribosomal DNA gene. J Clin Microbiol 1997; 35: 1216-1223.

[22] FELL JW, BOEKHOUT T, FONSECA A, SCORZETTI G, STATZELL-TALLMAN A. Biodiversity and systematics of basidiomycetous yeasts as determined by large-subunit rDNA D1/D2 domain sequence analysis. Int J Syst Evol Microbiol 2000; 50 Pt 3: 1351-1371.

[23] LI J, BAI F. Rapid identification of clinical yeast species by single-strand conformation polymorphism analysis of $26 \mathrm{~S} \mathrm{rDNA}$ D1/D2 domain. Wei Sheng Wu Xue Bao 2009; 49: 1011-1017.

[24] CHEN M, LIAO W, WU X. Sequence diversity of ribosomal internal transcribed spacer (ITS) region among Cryptococcus neoformans varieties. Chin J Dermatol 2007; 40: 230-233.

[25] DUPONT B. Use of topical antifungal agents. Therapie 2006; 61: 251-254. http://dx.doi.org/10.2515/therapie:2006041

[26] OHNEMUS U, WILLERS C, BUBENHEIM M, HORSTKOTTE MA, HOUDEK P et al. An ex-vivo oral mucosa infection model for the evaluation of the topical activity of antifungal agents. Mycoses 2008; 51: 21-29.

[27] BUTTICAZ G, ZULIAN GB, PREUMONT M, BUDTZ-JORGENSEN E. Evaluation of a nystatin-containing mouth rinse for terminally ill patients in palliative care. J Palliat Care 2003; 19: 95-99.

[28] DE PAUW B, WALSH TJ, DONNELLY JP, STEVENS DA, EDWARDS JE et al. Revised definitions of invasive fungal disease from the European Organization for Research and Treatment of Cancer/Invasive Fungal Infections Cooperative Group and the National Institute of Allergy and Infectious Diseases Mycoses Study Group (EORTC/MSG) Consensus Group. Clin Infect Dis 2008; 46: 1813-1821. http://dx.doi. org/10.1086/588660

[29] CORNELY OA, BOHME A, BUCHHEIDT D, EINSELE $\mathrm{H}$, HEINZ WJ et al. Primary prophylaxis of invasive fungal infections in patients with hematologic malignancies. Recommendations of the Infectious Diseases Working Party of the German Society for Haematology and Oncology. Haematologica 2009; 94: 113-122. http://dx.doi.org/10.3324/haematol.11665 